Revista de Derecho

\title{
Sentencia n. 9 904-12-JP/19 (negativa de atención en embarazo y violencia obstétrica)
}

\section{Case 904-12-JP/19 (pregnancy care denial and obstetric violencen)}

\section{Erick Fabián Guapizaca Jiménez*}

Asesor de despacho en la Corte Constitucional del Ecuador

Quito, Ecuador

erickguapizaca@hotmail.com

ORCID: 0000-0003-0263-9847

\section{María Paula Marroquín Ruiz*}

Oficial del Proyecto Justicia en Observatorio de Derechos y Justicia

Quito, Ecuador

mpmarroquinruiz@gmail.com

ORCID: 0000-0003-0320-7616

DOI: https://doi.org/10.32719/26312484.2021.35.5

Fecha de recepción: 29 de junio de 2020

Fecha de aceptación: 22 de septiembre de 2020

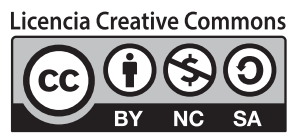

* Los autores agradecen profundamente los comentarios y sugerencias de los abogados Jean-Pierre Ruiz y Gustavo Villacreses, y de la comunicadora Diana Luzón. 


\section{RESUMEN}

El presente análisis jurisprudencial examina la sentencia 904-12-JP/19 (negativa de atención de embarazo y violencia obstétrica), de ponencia del juez Ramiro Ávila Santamaría, dictada por la Corte Constitucional del Ecuador. La sentencia fue dictada en el marco de la revisión de una sentencia de una acción de protección presentada por una mujer que no habría sido atendida adecuadamente por el sistema de maternidad del Instituto Ecuatoriano de Seguridad Social y que, producto de ello, sufrió actos de violencia obstétrica y vulneraciones a sus derechos a la salud y a la seguridad social. En una primera sección, el trabajo investigativo se describe y se analiza el proceso constitucional de selección y revisión de sentencias de garantías jurisdiccionales, así como el alcance y naturaleza de las sentencias de revisión. En una segunda sección, se estudia el peculiar método de la sentencia para introducir la problemática de la deficiente atención en salud a las mujeres embarazadas. Asimismo, se examina de manera crítica el razonamiento del juez respecto de la definición del contenido de los derechos que se declaran vulnerados: salud y seguridad social; se estudia la inédita incorporación del concepto de violencia obstétrica y se brinda un comentario respecto de las reparaciones ordenadas en el caso.

PALABRAS CLAVE: revisión, mujeres, embarazo, salud, violencia, obstétrica, seguridad, social.

\section{ABSTRACT}

This jurisprudential analysis examines judgment 904-12-JP/ 19 (denial to care for pregnancy and obstetric violence), delivered by Judge Ramiro Ávila Santamaría, issued by the Constitutional Court of Ecuador. The failure was handed down within the framework of the review of a sentence of a protection writ presented by a woman who had not been adequately cared for by the maternity system of the Ecuadorian Social Security Institute and who as a result suffered acts of obstetric violence and violations of their rights to health and to social security. In a first section, the investigative work describes and analyzes the constitutional process of selection and review of judgments of jurisdictional writs, as well as the scope and nature of the judgments of binding jurisprudence. In a second section, the peculiar method of the sentence is studied in detail to introduce the problem of deficient health care for pregnant women. Likewise, the reasoning of the judge regarding the definition of the content of the rights that are declared violated is critically examined: health and social security; The unprecedented incorporation of the concept of obstetric violence is studied and a comment is provided regarding the reparations ordered in the case.

KEYWORDs: review, women, pregnancy, health, violence, obstetric, security, social. 


\section{INTRODUCCIÓN}

T a salud reproductiva a la que tienen derecho las mujeres está relacionada con el Lejercicio de sus derechos a la salud, a la vida y a la integridad personal. Que el Estado no cumpla debidamente con su deber constitucional tiene graves consecuencias para las mujeres embarazadas. Por esta razón, la Constitución de la República del Ecuador (CRE), en su artículo 35, reconoce a las mujeres embarazadas como un grupo de atención prioritaria. ${ }^{1}$

De esta forma inicia el razonamiento del juez constitucional Ramiro Ávila, en la Sentencia n. ${ }^{\circ}$ 904-12-JP/19 dictada por la Corte Constitucional del Ecuador (CCE). De esta forma, el magistrado resalta el problema estructural que afrontan las mujeres embarazadas al momento de acceder a la atención prenatal, natal o postnatal dentro del sistema de seguridad social ecuatoriano. El fallo ilustra la necesidad de otorgar una mayor protección a los derechos de las mujeres y de imponer directrices claras para los prestadores de salud. En hechos como los que se examinarán a continuación, se determina que las mujeres son violentadas por parte del personal sanitario de las casas de salud que componen el sistema del Instituto Ecuatoriano de Seguridad Social (IESS).

Recientemente, la CCE se pronunció sobre un caso de violencia obstétrica en el contexto de la negativa de atención de una afiliada del IESS. El fallo dictado dentro del proceso de selección y revisión es inédito en cuanto ilustra cómo la CCE establece precedentes vinculantes respecto del contenido de los derechos y garantías consagrados en la CRE. Por ello, la pregunta transversal que atravesará este ensayo será cuál es el alcance de las sentencias de revisión.

En particular, la sentencia constituye una oportunidad crucial para la comprensión del contenido de los derechos a la salud, a la seguridad social y a la reparación integral. En virtud del razonamiento sobre el fondo del caso, la CCE se pronuncia sobre cada vulneración a estos derechos, otorgándoles un contenido esencial autónomo de los otros derechos constitucionales. Asimismo, esta sentencia desarrolló por primera vez, de manera jurisprudencial, el concepto de violencia obstétrica como resultado de estas graves violaciones a los derechos de las mujeres embarazadas.

El esquema para abordar lo planteado es: 1. El alcance que la CCE, la Ley Orgánica de Garantías Jurisdiccionales (LOGJCC) y la jurisprudencia constitucional le han dado a la facultad de revisión; 2. Los hechos del caso y el razonamiento judicial vertido en la sentencia analizada; y 3 . Las conclusiones.

1. Ecuador, Corte Constitucional, "Sentencia 904-12-JP/19", Caso n. ${ }^{\circ}$ 904-12-JP, 13 de diciembre de 2019. 


\section{LA FACULTAD DE REVISIÓN}

En la CRE se incorporó la competencia de la CCE para expedir jurisprudencia vinculante a partir de la revisión de sentencias de garantías jurisdiccionales seleccionadas por dicho organismo. ${ }^{2}$ Esta revisión versa sobre las sentencias resueltas por jueces ordinarios a nivel nacional en casos de garantías jurisdiccionales: acción de protección, hábeas data, acción de acceso a la información, hábeas corpus y medidas cautelares. ${ }^{3}$

La facultad de dictar decisiones vinculantes en materia constitucional es una innovación en el sistema ecuatoriano a partir de la actual Constitución y es importante por dos razones. Primero, es un mecanismo que permite la unificación en la aplicación de la norma constitucional. La consecuencia del carácter vinculante de los pronunciamientos, como en la revisión, es que estos tienen efectos erga omnes para todos los jueces que conozcan procesos de garantías jurisdiccionales y también como un auto precedente para la misma CCE. ${ }^{4}$ En segundo lugar, en el entendido que la CCE es el máximo órgano de administración de justicia en materia constitucional, permite la corrección de vulneraciones a derechos constitucionales que no fueron debidamente atendidas por los jueces ordinarios en los procesos de garantías jurisdiccionales. En la siguiente sección se estudiará el procedimiento de expedición de las sentencias que comúnmente se les conoce como de revisión.

\section{LAS ETAPAS DEL PROCEDIMIENTO DE REVISIÓN DE SENTENCIAS}

El proceso de selección y revisión se encuentra desarrollado en el artículo 25 de la LOGJCC. Esta norma estableció reglas para: 1. Los jueces ordinarios que conocen garantías jurisdiccionales; 2. Las Salas de Selección de la CCE; y 3. Las Salas de Revisión de la CCE.

La primera etapa obliga a todos los jueces ordinarios a remitir todas las sentencias ejecutoriadas que resuelven demandas de garantías jurisdiccionales a la CCE. ${ }^{5}$ Este primer momento habilita a la CCE a ejercer el rol de guardiana de la Constitución,

\footnotetext{
2. Ecuador, Constitución de la República del Ecuador, Registro Oficial 449, 20 de octubre de 2008, art. 436.

3. Agustín Grijalva, Constitucionalismo en Ecuador (Quito: Corte Constitucional para el Período de Transición, 2011), 183-7.

4. Pamela Aguirre, "El valor de la jurisprudencia dentro del Ordenamiento Constitucional Ecuatoriano ¿Cambio de paradigma?", Iurisprudentia Revista da Facultade de Direito 6, n. ${ }^{\circ} 12$ (2017): 122-3, https:// bit.ly/3g07Zsi.

5. Carlos Alberto Agudelo, "Diálogos Internos en la Corte Constitucional Colombiana sobre el modus ope-
} 
al poder examinar la forma en la que los operadores de justicia ordinarios aplican la norma constitucional en la tramitación los procesos constitucionales.

Posterior a la remisión de sentencias, corresponde a la Sala de Selección de la $\mathrm{CCE}$, integrada por tres jueces constitucionales,${ }^{6}$ escoger de forma "discrecional" las sentencias sobre las que se desarrollará la jurisprudencia vinculante. Ahora bien, es oportuno puntualizar que, si bien la Sala de Selección tiene discrecionalidad en la selección en su motivación, se deben observar cuatro parámetros:

a) Gravedad del asunto,

b) Novedad del caso e inexistencia de precedente judicial,

c) Negación de los precedentes judiciales fijados por la Corte Constitucional y

d) Relevancia o trascendencia nacional del asunto resuelto en la sentencia. ${ }^{7}$

La LOGJCC no se refiere a si los parámetros citados deben cumplirse de forma concurrente o si basta con que se justifique uno para la selección de una sentencia. No obstante, se ha establecido que se debe poner especial atención en el parámetro de gravedad del asunto, puesto que se debe dar prioridad a aquellos casos que, por la intensidad de la vulneración, ameriten una reparación. ${ }^{8}$

Un tercer momento se encuentra a cargo de la Sala de Revisión integrada por tres jueces de la CCE cuya obligación es aprobar un proyecto de sentencia en la que se establezca jurisprudencia vinculante respecto de los hechos de la sentencia revisada. El proyecto debe ser elaborado por uno de los magistrados y a los dos restantes les compete aprobar o rechazar el mismo; con dos votos a favor, el proyecto es aprobado. Si embargo, se destaca que el proceso no concluye formalmente en la Sala de Revisión pues después se debe remitir el proyecto de sentencia al Pleno de la CCE para su discusión y aprobación. ${ }^{9}$ Para la aprobación del proyecto se requieren de cinco votos afirmativos de entre los nueve jueces constitucionales.

randi en la selección de tutela entre la informalidad, la política y la academia", Jurídicas (Universidad de Caldas) 7, n. ${ }^{\circ} 2$ (2010): 58, https://bit.ly/2Ae79ZQ.

6. Ecuador, Reglamento de Sustanciación de Procesos de Competencia de la Corte Constitucional, Registro Oficial, Suplemento 613, 22 de octubre de 2015, art. 25.

7. Ecuador, Ley Orgánica de Garantías Jurisdiccionales y Control Constitucional, Registro Oficial, Suplemento, 22 de octubre de 2019, art. 25.

8. Juan Montaña Pinto, "Aproximación a los elementos básicos de la acción de protección”, en Apuntes de derecho procesal constitucional, eds. Juan Montaña Pinto y Angélica Porras Velasco (Quito: Corte Constitucional para el Período de Transición / Centro de Estudios de Difusión del Derecho Constitucional Ecuatoriano, 2012), 111.

9. Ecuador, Reglamento de Sustanciación de Procesos de Competencia de la Corte Constitucional, art. 27. 


\section{ALCANCE DE UNA SENTENCIA DE REVISIÓN}

Para responder la pregunta introductoria se debe retomar el propósito principal de la facultad de revisión, unificar fallos y crear precedentes vinculantes en materia constitucional. Por ello, se podría discutir si la CCE puede pronunciarse sobre: 1. Las vulneraciones a derechos identificadas en el caso, dictar una sentencia sobre el fondo y definir el contenido de estos y de las garantías jurisdiccionales; o si, por el contrario, 2. Solo puede delimitar las reglas de tramitación de las garantías jurisdiccionales sin alterar lo ya decidido por los jueces de instancia. ${ }^{10}$

La primera decisión de revisión de garantías jurisdiccionales abordó esta cuestión. En la sentencia n. ${ }^{\circ}$ 001-PJO-CC denominada "Indulac" se habilitó a la CCE para que examine las pretensiones de las partes, declare vulneraciones por parte de los jueces que conocieron la causa y repare a las víctimas, en los siguientes términos:

Está claro que si durante el proceso de desarrollo de jurisprudencia vinculante se identifican en el caso materia de estudio vulneraciones a derechos constitucionales, la Corte Constitucional se encuentra plenamente facultada, a través de la revisión del caso, a reparar las consecuencias de dicha vulneración. ${ }^{11}$

Para Emilio Suárez, esta sentencia, hito en materia de revisión, distorsionó la naturaleza de la facultad de revisión al transformarla en una garantía jurisdiccional más e incluso en una instancia de apelación en materia constitucional. Bajo esta consideración, para el autor, el diseño original de la facultad analizada no permite que la CCE se pronuncie sobre el fondo del asunto, sino que se debe limitar a "dicta[r] precedentes vinculantes que se incorporan al ordenamiento jurídico. Esto descarta la posibilidad de considerarla como un recurso o una acción". ${ }^{12}$ Inclusive, para esta posición de la doctrina habría una duplicación en las funciones de tutela del organismo de control constitucional debido a que la acción extraordinaria de protección sería la única vía adecuada para la protección de los derechos constitucionales. ${ }^{13}$

Respecto a este criterio se puntualiza que el rol de la Corte Constitucional, como la guardiana de la CRE, tendría una facultad débil, incompleta y hasta contradictoria en

10. Paúl Córdova Vinueza, Derecho Procesal Constitucional (Quito: Corporación de Estudios y Publicaciones, 2016), 229.

11. Ecuador, Corte Constitucional, "Sentencia 001-PJO-CC", Caso n. ${ }^{\circ}$ 0999-09-JP, 22 de diciembre de 2010, párrs. 19-23.

12. Emilio Suárez, "Distorsiones del sistema de selección y revisión de sentencias de la Corte Constitucional Ecuatoriana" (Programa Maestría en Derecho Mención en Derecho Constitucional, Universidad Andina Simón Bolívar, Sede Ecuador, 2015), 87.

13. Córdova Vinueza, Derechos Procesal Constitucional, 231-2. 
aquellos casos en que dicho órgano se limite a sentar precedentes sobre el funcionamiento de las garantías jurisdiccionales sin pronunciarse sobre el fondo del caso. Esto específicamente para aquellos casos en que se verifique que no se tutelaron adecuadamente derechos constitucionales y que por tanto ameriten medidas de reparación.

En este sentido, no solo interpretaría el contenido de la CRE, sino que obliga a su cumplimiento y directa aplicación en aquellas circunstancias en que cualquier persona, entidad pública o judicial la haya inobservado. En ello se debe enfatizar que la jurisprudencia constitucional "es un acto creador de derecho" o, en otras palabras, una fuente de derecho a ser observada por los operadores de justicia en la resolución de las garantías jurisdiccionales y por las entidades públicas dentro del marco de sus competencias. ${ }^{14}$

No obstante, un claro límite del precedente estudiado es que no toma en cuenta los argumentos que podrían tener las entidades o personas demandadas y tampoco considera la situación actual de las personas afectadas. Años más tarde, la Sentencia n. ${ }^{\circ}$ 159-11-JH/19 ratificó el criterio aplicable para fallos de revisión que permitía reabrir el fondo y pronunciarse sobre las violaciones a derechos constitucionales alegadas. En esta ocasión el juez Ávila se pronunció sobre la privación de libertad de una persona en situación de movilidad y dio un peculiar revés al trámite de los casos de revisión. En este fallo se reconoció la inmensa carga procesal con la que contaba la CCE, situación que impedía la tramitación rápida de la mayoría de causas. Por ello, declaró la inconstitucionalidad sobre la regla establecida en el artículo 25 número 6 de la LOGJCC que prescribe que las causas no han sido seleccionadas, dentro del término de 20 días desde su recepción, se entienden automáticamente excluidas de la revisión. En su razonamiento, el Pleno de la CCE determinó que esta norma afecta innecesariamente los derechos a la tutela judicial efectiva y a la reparación integral en vigor de que no habilita a la CCE el establecimiento de medidas de reparación en casos en los que las afectaciones, a pesar del transcurso del tiempo, aún subsisten. ${ }^{15}$

La resolución judicial aludida también abordó la preocupación respecto de la defensa de las entidades demandas en cuanto a que sería oportuno citar a las partes procesales a las que pueda alcanzar el contenido de la decisión para que presenten sus argumentos y puedan ejercer el derecho a la contradicción. ${ }^{16}$ La CCE determinó que se debe notificar a todas las partes procesales desde el avoco de conocimiento de las causas seleccionadas, convocar a una audiencia en que las partes sean debidamente

14. Hans Kelsen, Teoría Pura del Derecho, trad. Roberto Verengo (Ciudad de México: Porrúa, 2009$), 152$.

15. Ecuador, Corte Constitucional, "Sentencia n. ${ }^{\circ}$ 001-14-PJO-CC", Caso n. ${ }^{\circ}$ 001-14-PJO-CC, 23 de abril de 2014.

16. Julio César Trujillo, Constitucionalismo contemporáneo: teoría, procesos, procedimientos y retos (Quito: Corporación Editora Nacional / Universidad Andina Simón Bolívar, Sede Ecuador, 2013), 287. 
escuchadas y restringir el análisis jurídico a los hechos del caso que han sido conocidos en instancia. ${ }^{17}$

La CCE ha analizado tanto las actuaciones de los jueces que conocieron las garantías jurisdiccionales, así como aquellas de las entidades demandadas. Esto se plasmó en la motivación de la Sentencia n. ${ }^{\circ} 159-11-\mathrm{JH} / 19$, que se declaró vulneraciones por parte de los jueces que conocieron la acción de hábeas corpus, así como del personal del Servicio Migratorio que detuvo a la persona en movilidad humana. ${ }^{18}$ Con un alcance similar, la sentencia del caso "Diario La Hora" desarrolló el contenido del derecho a la libertad de expresión como un pilar fundamental para una sociedad democrática. ${ }^{19}$

Por otro lado, en el fallo n. ${ }^{\circ} 603-12-J P / 19$ se resolvió de manera distinta, muy importante porque ratificó el criterio vertido por el juez de instancia, lo que conllevó a que únicamente establezca estándares a ser tomados en cuenta por parte de los funcionarios del Registro Civil al momento de registrar uniones de hecho de personas del mismo sexo. ${ }^{20}$ En relación con lo analizado, se considera necesario aclarar que, de acuerdo con el mismo texto de la Constitución, todo el razonamiento de las sentencias de la CCE debe ser tomado en cuenta de forma obligatoria por parte de todos los jueces. ${ }^{21}$

Las decisiones citadas anteriormente tienen un denominador común (a excepción de la Sentencia n..$^{\circ}$ 603-12-JP/19): la mayoría se refiere a cuál es la interpretación que tienen las distintas garantías jurisdiccionales y, en ciertos pronunciamientos, como los que se refieren a la movilidad humana y a la libertad de expresión, llegan a delimitar el contenido de ciertas normas constitucionales.

De lo mencionado anteriormente se desprende que no es una regla general reabrir todos los procesos nuevamente y pronunciarse respecto del fondo, pues dependerá del caso. Esto porque dicho organismo constitucional no necesariamente selecciona los casos en los que se pretenda reparar vulneraciones a derechos, sino también aquellos en los que los jueces ordinarios hayan tutelado oportunamente los derechos de los accionantes, con el objetivo de ampliar ciertos conceptos y desarrollar reglas vinculantes.

Por las consideraciones desarrolladas en esta sección, el fallo del caso n. ${ }^{\circ} 492-$ 12-JP/19 resulta relevante para comprender la configuración actual del alcance de la facultad de revisión de la Corte Constitucional. Esto, principalmente dado que no establece estándares para el uso de la acción de protección, sino que desarrolla el

17. Ecuador, Corte Constitucional, "Sentencia 159-11-JH/19”, Caso n. ${ }^{\circ} 159-11-J H, 26$ de noviembre de 2019.

18. Ibíd.

19. Ecuador, Corte Constitucional, "Sentencia 282-13-JP/19”, Caso n. ${ }^{\circ} 282-13-J P, 4$ de septiembre de 2019.

20. Ecuador, Corte Constitucional, "Sentencia 603-12-JP/19", Caso n. ${ }^{\circ}$ 603-12-JP, 5 de noviembre de 2019.

21. Ecuador, Constitución de la República del Ecuador, art. 436. 
contenido de varios derechos constitucionales, ratifica su violación y amplía las reparaciones ordenadas por el tribunal que expidió la sentencia de revisión.

\section{EL CASO NOLE OCHOA}

La sentencia aborda las vulneraciones a los derechos constitucionales de la señora Jessika del Rosario Nole Ochoa (la accionante), una trabajadora de una compañía privada, quien, al momento que ocurrieron los hechos del caso, se encontraba afiliada al IESS. En mayo de 2011, ella ingresó al Hospital del Seguro Social de la ciudad de Machala (HSSCM) en horas de la tarde. Conforme los dolores aumentaron, ella gritaba de dolor sin que el personal del referido hospital le prestara atención. En el recuento de los hechos se precisa que el bebé nació sin asistencia médica alguna.

Como consecuencia del parto, el útero de la accionante se habría desagarrado y sufrido un cuadro de hemorragia. Luego de ello, el personal médico del IESS le informó que debían trasladarle a otra casa de salud pública dado que ella presuntamente no tenía vigente el derecho de atención médica, ello justificado en que la señora supuestamente adeudaba aportes al sistema de seguridad social. Al salir del hospital público al que fue trasladada, solicitó su historia clínica y una explicación por el rechazo de atención por parte del Hospital del IESS. Inconforme con la información que recibió, y al encontrar que estaba incompleta, solicitó por segunda ocasión que le otorguen una copia de su historial médico completo. Después presentó una acción de protección en contra de la Dirección Provincial del IESS.

En primera instancia, la actora obtuvo una sentencia favorable que declaró la vulneración a sus derechos constitucionales a la atención médica preferente y a la atención del recién nacido. Como medidas de reparación integral, la jueza que conoció la causa ordenó a las entidades demandadas que reparen económicamente a la accionante, presenten disculpas públicas y que la casa de salud que la expulsó se comprometa a que no vuelvan a ocurrir este tipo de casos. La entidad demandada interpuso recurso de apelación.

En segunda instancia, el tribunal que conoció la causa confirmó la sentencia y revocó la medida de disculpas públicas precisando que esta fue ordenada a una institución ajena al proceso de acción de protección. La decisión de segunda instancia fue remitida a la CCE, donde la Sala de Selección resolvió escoger el caso de la señora Nole Ochoa para el desarrollo de jurisprudencia vinculante. A continuación, se exa- 
minará el razonamiento de la CCE respecto de las vulneraciones a derechos alegadas por la accionante. ${ }^{22}$

\section{RAZONAMIENTO DE LA CORTE}

El esquema de la sentencia, primero, tiene como eje central un apartado de "consideraciones previas" que ilustra el problema en el que se enmarcan los hechos. A continuación, se explica el marco de protección aplicable, incluyendo las normas constitucionales e instrumentos internacionales en derechos humanos aplicables al caso. Y, finalmente, se subsume los estándares a los hechos.

Como se lo anticipó, un acierto que es transversal a toda la decisión es que el juez presenta de manera objetiva el contexto de la problemática respecto al acceso a la salud de las mujeres embarazadas en el apartado de "consideraciones previas". Presenta estadísticas que grafican la incidencia de mortalidad materna e infantil y enuncia recomendaciones de organismos internaciones para prevenir la violencia obstétrica. Esta forma de introducir las problemáticas es inédita desde la integración del magistrado Ávila a la Corte Constitucional.

Por ejemplo, la decisión que resolvió la consulta de norma respecto de la aplicación de Opinión Consultiva OC-24/17 es uno de los casos que incluye en su análisis constitucional datos y cifras que reflejan problemas que afectan la vigencia plena de derechos constitucionales. En dicho fallo se declaró que la interpretación respecto del art. 67 de la CRE es aquella que permite el matrimonio sin discriminación del sexo u orientación de las personas. Para llegar a su conclusión, el juez constitucional incluyó datos estadísticos respecto de "la situación de las personas con identidades sexogenéricas diversas". ${ }^{23}$ También lo ha hecho en la Sentencia n. ${ }^{\circ} 159-11-\mathrm{JH} / 19$, que presentó antecedentes en relación a los problemas que afrontan los migrantes que ingresan al territorio ecuatoriano.

Además, se destaca que el juez buscó dar protagonismo a la víctima en su sentencia, puesto que, en varias de sus secciones, incorpora la voz de la persona afectada, por ejemplo, con las continuas citas de lo dicho durante la audiencia. Esta concepción la replicó de su libro La utopía del oprimido que cuestiona si “¿[puede] la Cosmovisión de los pueblos y las luchas sociales abrevar la política, el derecho, el pensamiento

22. Ecuador, Ley Orgánica de Garantías Jurisdiccionales y Control Constitucional, art. 170.

23. Ecuador, Corte Constitucional, "Sentencia 11-18-CN/19", Caso n. ${ }^{\circ} 11-18-C N / 19$, 12 de junio de 2019 , párr. 300. 
crítico?". ${ }^{24}$ Como respuesta, el magistrado considera que la justicia debe representar una respuesta a las luchas sociales.

Otro aspecto general sobre el esquema que podría pasar desapercibido es que no se presenta la formulación de problemas jurídicos, como acostumbran las sentencias de la Corte Constitucional, mediante preguntas. Ahora bien, un problema jurídico desde la perspectiva de Theodor Viehweg se caracteriza por ser toda cuestión que en apariencia tiene una sola respuesta única y que necesita argumentos para su resolución. ${ }^{25}$

Conforme lo reseñado, no es necesario que se plantee el problema jurídico a modo de pregunta pues bastará explicar, grosso modo, la situación a analizar. Esto se refuerza con lo desarrollado por Manuel Atienza, para quien los problemas jurídicos deberían tener "una naturaleza bivalente: aceptar o no un recurso, anular o no una norma". ${ }^{26}$ Lo que sí se cumple en la sentencia dado que se busca determinar si se vulneró o no un derecho constitucional.

Otro aspecto estructural de la sentencia es respecto de su motivación y análisis sobre las vulneraciones a derechos identificadas. Como corolario, todos los jueces se encuentran obligados a motivar una sentencia; este ejercicio consiste en determinar con base en la normativa aplicable, en este caso la CRE y los instrumentos internacionales, si se encontraron o no vulneraciones a derechos. ${ }^{27}$ Este ejercicio se ha cristalizado en el ordenamiento jurídico como una garantía del debido proceso que es obligación no solo de los jueces al momento de dictar sentencia, sino también de toda persona que emita una resolución pública. A continuación se realizará un estudio crítico de la motivación del fallo respecto de los derechos a la salud, a la seguridad social y a la reparación integral.

\section{RESPECTO DEL DERECHO A LA SALUD}

La sentencia en sus consideraciones previas presenta estadísticas del problema de la deficiente atención que enfrentan las mujeres embarazadas por parte de las casas de salud que integran el IESS. La decisión advierte que esta atención deficiente podría vulnerar otros derechos. Precisamente, uno de los principales debates en los sistemas

24. Ramiro Ávila Santamaría, La utopía del oprimido: Los derechos de la naturaleza y el buen vivir en el pensamiento crítico, el derecho y la literatura (Ciudad de México: Akal, 2019), 6.

25. Ramón Beltrán Calfurrapa, "La tópica jurídica y su vinculación argumentativa con el precedente y la jurisprudencia", Revista de derecho a Pontificia Universidad Católica de Valparaíso, n. ${ }^{\circ} 39$ (2012): 587-8, $10.4067 / \mathrm{S} 0718-68512012000200021$.

26. Manuel Atienza, Curso de argumentación jurídica (Madrid: Trotta, 2013), 90.

27. Ernesto Galindo Sifuentes, Argumentación jurídica: Técnicas de argumentación del abogado y del juez (Ciudad de México: Porrúa, 2013), 30-2. 
de protección de derechos humanos es el que atañe a la justiciabilidad de los derechos económicos, sociales y culturales.

El derecho a la salud no ha sido exento de esta disyuntiva. Tanto es así que en el año 2018 la Corte Interamericana de Derechos Humanos (Corte IDH) dirimió por primera vez este debate en la sentencia del caso Poblete Vilches c. Chile, al pronunciarse sobre la vulneración de este directamente. ${ }^{28}$ Anteriormente el análisis de la Corte IDH sobre este asunto siempre se encontraba atado a que se declaren violaciones a derechos civiles y políticos, como la vida e integridad física. ${ }^{29}$

Retomando a la sentencia en análisis, se debe precisar que la explicación del alcance de este derecho da cuenta del carácter autónomo y plenamente justiciable con el que se lo abordó en la jurisprudencia constitucional ecuatoriana. "No sorprende esta característica, puesto que la CCE ha señalado que los derechos contenidos en ella gozan de igual jerarquía que la división entre primera, segunda y tercera generación ha sido superada".$^{30}$

Llama también la atención el uso del estándar de los elementos del derecho a la salud del Comité de Derechos Económicos Sociales y Culturales (Desc) "disponibilidad, accesibilidad, aceptabilidad y calidad". ${ }^{11}$ Ello dado que, por primera vez, se emplean estos elementos como ratio decidendi ${ }^{32}$ al subsumir cada uno de estos al caso de la accionante y, con base en el incumplimiento de estos, concluir que la desatención de su parto vulneró su derecho a la salud. Antes se había empleado de manera complementaria los elementos para definir el contenido mínimo del derecho estudiado. ${ }^{33}$

En este contexto, se destaca la inclinación de la CCE en incluir en el bloque de constitucionalidad a todos los instrumentos de Derechos Humanos y no solo a tratados. Esta interpretación de la Constitución se ha visto aplicada en diversas sentencias, destacándose la Sentencia n. ${ }^{\circ}$ 184-18- SEP- CC, mejor conocida como el "Caso Satya", ${ }^{34}$ y la citada Sentencia n. ${ }^{\circ} 11-18-\mathrm{CN} .{ }^{35}$

28. Corte IDH, "Sentencia de 8 de marzo de 2018 (Excepciones Preliminares, Fondo, Reparaciones y Costas)", Caso Poblete Vilches vs. Ecuador, 8 de marzo de 2018, párr. 108, https://bit.ly/3dGIHOi.

29. Patricio Pazmiño Freire, "El Derecho a la salud y la especial protección de las personas con VIH. Desarrollo jurisprudencial y desafíos del acceso directo, progresividad y la reparación", Oficina del Alto Comisionado para los Derechos Humanos, 13 de febrero de 2019, https://bit.ly/3dEsERc.

30. Ecuador, Corte Constitucional, "Sentencia n. ${ }^{\circ}$ 016-16-SEP-CC", en Caso n. ${ }^{\circ}$ 2014-12-EP, 13 de enero de 2016.

31. ONU Comité de Derechos Económicos, Sociales y Culturales, Observación General $n .^{\circ} 19$. El derecho a la seguridad social articulo 9, 4 de febrero de 2018, E/C.12/GC/19.

32. Entendida como la razón de la decisión.

33. Ibíd.

34. Ecuador, Corte Constitucional, "Sentencia 184-18-SEP-CC", Caso n. ${ }^{\circ} 1692-12-E P, 29$ de mayo de 2018.

35. Ecuador, Corte Constitucional, "Sentencia 11-18-CN/19", Caso n. ${ }^{\circ} 11-18-C N / 19,12$ de junio de 2019, párr. 300. 
No obstante, sobre los cuatro elementos del derecho a la salud, se debe señalar que la aproximación del Comité de Desc sobre el derecho a la salud podría tener ciertos límites, pues, plantearlo con los elementos citados, conlleva a reducirlo a un problema de hospitales, lo que dejaría de lado otras obligaciones incluidas en el contenido esencial del derecho tales como las políticas de prevención de enfermedades, el manejo de residuos y la contaminación ambiental. ${ }^{36}$

\section{ACERCA DE LA VIOLENCIA OBSTÉTRICA}

En un acápite separado del concerniente al derecho a la salud bien hace en explicar cómo la negativa de atención a la accionante es un ejemplo de maltrato que hace que las personas dejen de utilizar los servicios públicos de salud. ${ }^{37}$ En este punto se aborda el problema estructural de la deficiente atención materna prestada por la casa de salud demandada y de las consecuencias que acarrearon en el derecho a la salud de la accionante.

$\mathrm{Al}$ respecto, la sentencia reconoce que "la violencia contra la mujer se manifiesta en múltiples escenarios, uno de ellos ocurre en relación a la prestación de servicios y atención de salud, en ese contexto un tipo específico de violencia es la obstétrica". ${ }^{38}$

En concreto, uno de los puntos más fuertes del razonamiento de la Corte para argumentar una transgresión de los derechos a la salud de la afectada es el ateniente a la violencia obstétrica. Es importante destacar que el fallo logró incorporar de forma inédita un concepto que impone límites claros y específicos a los profesionales de la salud que atienden a mujeres embarazadas. Así, la incorporación de este concepto en la sentencia tiene efectos prácticos, dado que ejemplifica ciertas acciones u omisiones que pueden recaer en violencia obstétrica ${ }^{39} \mathrm{y}$ para ello se fundamenta directamente en la Declaración sobre la Eliminación de la Violencia contra la Mujer de las Naciones Unidas, la Convención Belém do Pará y en el informe de la Relatora de las Naciones Unidas sobre la violencia para la Mujer, lo que además demuestra un logrado control convencional por parte de la CCE. ${ }^{40}$

36. Víctor Currea-Lugo, La salud como derecho humano (Bilbao: Universidad Deusto, 2005), 75-6.

37. Ecuador, Corte Constitucional, "Sentencia 904-12-JP/19".

38. Ibíd.

39. Josefina Goberna-Tricas, "Violencia obstétrica: aproximación al concepto y debate en relación a la terminología empleada", MUSAS. Revista de Investigación en Mujer, Salud y Sociedad 4, n. ${ }^{\circ}$ (2019): 26, doi: 10.1344/musas2019.vol4.num2.2.

40. Ecuador, Constitución, art. 155. 
Se resalta que el concepto desarrollado se aplicó a los hechos del caso pues se concluyó que en el caso concreto, por ejemplo, el abandono por parte del personal sanitario durante el nacimiento del bebé devino en afectaciones a su derecho a la salud. ${ }^{41}$ No obstante, resulta un límite de la sentencia el siguiente razonamiento: "la violencia obstétrica sufrida constituye una violación a su derecho a la salud en conexidad con otros derechos", ${ }^{42}$ dado que los hechos del caso permitían que se analice también la transgresión del derecho a una vida libre de violencia en el ámbito público y privado, cuyo contenido esencial no ha sido desarrollado por la CCE. ${ }^{43}$

\section{SOBRE EL DERECHO A LA SEGURIDAD SOCIAL}

La seguridad social, en su dimensión como un derecho humano, busca mitigar los efectos de la exclusión económica y social de los más vulnerables, dando mayor certeza a los individuos de una vida digna en la que se promuevan y garanticen sus derechos. ${ }^{44}$ Uno de los servicios de los sistemas de seguridad social es el de asistencia por maternidad.

Un problema central que trata el fallo es referente a la deficiente atención por maternidad que brindó el HSSCM a la accionante. Es notable de la sentencia hace, de forma inédita, una mención sobre que las prestaciones de salud a la mujer deben ser apropiadas "durante el período prenatal, durante el parto y en el período posnatal, que incluye la hospitalización" ${ }^{45}$ y enfatiza el hecho de que sí existió la atención, pero no fue la apropiada.

Adicionalmente, es importante la precisión de que la actuación del HSCM fue arbitraria pues "exigió para tener derecho a la cobertura, estar al día con el pago de contribuciones patronales" ${ }^{46} \mathrm{El} \mathrm{juez} \mathrm{critica} \mathrm{fuertemente} \mathrm{la} \mathrm{constancia} \mathrm{de} \mathrm{que} \mathrm{la} \mathrm{señora}$ accionante "no poseía vigencia del derecho", de acuerdo con el personal del hospital. Ello, por una parte, debido a la pertenencia de la mujer embarazada a un grupo de atención prioritaria y, por otra, a la imposibilidad de condicionar un derecho de salud por compromiso económico. Es interesante que el organismo de control constitucional indica que este aspecto se encuentra desarrollado en la ley ecuatoriana pues da

41. Ecuador, Corte Constitucional, "Sentencia 904-12-JP/19".

42. Ibíd.

43. Ecuador, Constitución, art. 66 núm. 3 literal b.

44. ONU Comité de Derechos Económicos, Sociales y Culturales, Observación General n. ${ }^{\circ}$ 19. El derecho a la seguridad social artículo 9, 4 de febrero de 2018, E/C.12/GC/19.

45. Ecuador, Corte Constitucional, "Sentencia 904-12-JP/19".

46. Ibíd. 
cuenta del desarrollo progresivo del derecho a la seguridad social al implementarse por vía de ley disposiciones que prohíban restricciones ilegítimas.

Más allá de esto, la Corte aclara que no se puede limitar el ejercicio de los derechos de salud de las mujeres embarazadas a ningún compromiso económico, como el ejemplo del caso concreto de la supuesta mora patronal. Por lo tanto, incorpora un avance muy claro con respecto al derecho de seguridad social al dictaminar que este grupo de atención prioritaria no podrá ser restringido en ningún ámbito de las prestaciones que presta el IESS a sus asegurados.

Una crítica que merece la sentencia es sobre la carencia de un análisis interdependiente entre el derecho a la seguridad social y a la salud. La CRE consagró el principio de interdependencia en su art. 11 núm. $6 .{ }^{47}$ Este principio ha sido definido como aquel que señala la medida en que el disfrute de un derecho en particular o un grupo de derechos dependen para su existencia de la realización de otro derecho. ${ }^{48}$

Por consiguiente, si bien de la sentencia se destaca que el juez ponente buscó darle personalidad propia a cada derecho un examen más fuerte, hubiera implicado tomar en cuenta las consecuencias directas que tuvo la arbitraria privación del derecho a la seguridad social para el menoscabo al derecho a la salud de la accionante.

\section{SOBRE EL DERECHO LA REPARACIÓN INTEGRAL}

De acuerdo con el núm. 3 del art. 86 de la Constitución, un deber correlativo al haberse declarado vulneración de derechos es repararlos. ${ }^{49}$ Por esto, la actual magistrada de la Corte Constitucional Karla Andrade Quevedo explica que "solo la reparación integral garantiza que la acción de protección cumpla de lleno su objeto y garantice la eficacia y supremacía de los derechos constitucionales". ${ }^{50}$

En el fallo, las medidas ordenadas al IESS consistieron en: la compensación económica por los gastos incurridos al no ser atendida oportunamente, el pago en equidad de $\$ 5000$ por el daño inmaterial producido por la violencia obstétrica y, como medida de satisfacción, se ordenó la difusión de las disculpas públicas y la difusión de la

47. Ecuador, Constitución, art. 11, núm. 6.

48. Sandra Serrano y Luis Daniel Vázquez, "Los principios de universalidad, interdependencia, indivisibilidad y progresividad. Apuntes para su aplicación práctica”, Biblioteca Jurídica Virtual del Instituto de Investigaciones jurídicas de la UNAM, https://bit.ly/3g1U7xI.

49. Ecuador, Constitución, art. 86, núm. 3.

50. Karla Andrade, "La acción de protección en la jurisprudencia de la Corte", en Manual de justicia constitucional ecuatoriana, eds. Jorge Benavides Ordóñez y Jhoel Escudero Soliz (Quito: Corte Constitucional / Centro de Estudios y Difusión, 2013), 111. 
sentencia en el portal web del IESS. En lo que atañe al caso de la accionante, se debe puntualizar que no era posible la restitución de los derechos a la salud y a la seguridad social y devolver a la accionante al estado anterior a su vulneración. ${ }^{51}$ Para la doctrina constitucional devolver la situación al estado anterior "constituye el máximo ideal de la reparación integral, por cuanto define que devolver a la víctima a su estado anterior conforma la finalidad óptima de alcanzar la reparación integral". ${ }^{52}$

A pesar de ello, es un acierto el conjunto de medidas ordenadas para intentar satisfacer las violaciones identificadas, sobre todo la difusión de la decisión y la publicación de disculpas públicas. Además de ello, se debe destacar que se determinó en "equidad" el monto económico a pagar a la afectada. En este contexto, se observa que, para la actual composición de la Corte Constitucional, el monto a pagar por el daño a los derechos puede cuantificarse sin la necesidad de "erogaciones monetarias y aun en esencia de todos los recibos necesarios". ${ }^{53}$ A primera vista, este ejercicio podría parecer arbitrario para la parte accionada. Sin embargo, dado que, en el caso las vulneraciones fueron verificadas por la CCE, se procedió a analizar objetivamente los gastos en los que la accionante pudo haber incurrido por gastos médicos y se calculó con base en ello el monto indicado. ${ }^{54}$

$\mathrm{Al}$ declararse vulneraciones a los derechos constitucionales a la salud y seguridad social, la CCE ordenó medidas para garantizar la protección del derecho a un nivel más amplio y para evitar que vuelvan a ocurrir hechos como los examinados. Esto ocurre específicamente en las medidas de no repetición que ordenan al IESS como obligado principal a realizar una "guía integral de atención a las mujeres embarazadas y de prevención de la violencia obstétrica" y a "efectuar un plan de revisión técnico a nivel nacional para verificar que los establecimientos de salud públicos y privados cuenten con las condiciones necesarias [...] para asegurar que los derechos de salud de las mujeres embarazadas se vean garantizados". Se debe destacar que esta medida de reparación busca incidir directamente en el ejercicio de los derechos indicados y a tomar en cuenta la obligación de trato prioritario.

51. Corte IDH, "Sentencia de 21 de julio de 1989 (Excepciones Preliminares, Fondo, Reparaciones y Costas)", Caso Velázquez Rodríguez vs. Honduras, 21 de julio de 1989, párr. 189, https://bit.ly/2ZhL5FV.

52. Pamela Aguirre Castro y Pablo Alarcón, "El Estándar de la Reparación Integral en la Jurisprudencia de la Corte Constitucional", FORO: Revista de Derecho, n. ${ }^{\circ} 30$ (2018), doi: 10.32719/26312484.2018.30.8.

53. Graciela Rodríguez Manzo et al., Responsabilidad y reparación en enfoque de Derechos Humanos (Ciudad de México: Comisión de Derechos Humanos del Distrito Federal / Universidad Iberoamericana, 2007), 148.

54. Corte IDH, "Sentencia de 1 de julio de 2016 (Excepciones Preliminares, Fondo, Reparaciones y Costas)", Caso de las Masacres de Ituango vs. Colombia, 1 de julio de 2016, párr. 379, https://bit.ly/3dLxCf7. 


\section{CONCLUSIONES}

En respuesta al planteamiento inicial acerca de cuál es el alcance de las sentencias de revisión, el fallo comentado ha sido bastante ilustrativo para la comprensión de importancia, alcance y poder transformador que tiene la CCE al momento de ejercer su facultad de revisión. Así, en el caso concreto se volvieron a examinar los hechos del caso, se declararon más vulneraciones que la sentencia revisada y se desarrollaron reparaciones tanto para el caso concreto como medidas de no repetición ordenadas para evitar la ocurrencia de nuevos actos de violencia obstétrica.

Además, en el desarrollo de la investigación se rescató la forma en la que el juez sustanciador coloca sobre la mesa los problemas jurídicos. Esto ocurre puesto que no deja entrever que lo sucedido a la accionante sea un caso aislado, sino que se enmarca en un problema estructural en el sistema de salud ecuatoriano. A partir de ello procede a motivar las vulneraciones identificadas en el caso incorporando, con un control de convencionalidad directo, varios estándares en materia de derechos humanos. Este aspecto también permitió corroborar que se busca incluir a la jurisprudencia constitucional a la par de un orden mundial en materia de protección de derechos.

En relación con el derecho a la salud, se destacó la manera en que la CCE examina su vulneración a partir de la recepción de la Observación General n. ${ }^{\circ}$ 14, comprobando el cumplimiento o no de los elementos de dicho instrumento. ${ }^{55}$ Por otro lado, se persistió en la preocupación de que la CCE limitó el ejercicio del derecho a la salud a un problema en los servicios hospitalarios, pues se debería observar también otros ámbitos importantes para el ejercicio de este derecho fundamental.

El pronunciamiento sobre el derecho a la seguridad social fue analizado respecto de la negativa de acceso por mora patronal. Este punto es destacable del razonamiento del juez pues es compatible con el objeto de la seguridad social: mitigar los efectos de la exclusión social. No obstante, se criticó la omisión de la CCE al no analizar la vulneración de manera interdependiente al derecho a la salud. Lo que podría haber dado un panorama más amplió de las deficiencias en los sistemas de salud prestados en el sistema de seguridad social. Sin lugar a duda, los hechos sucedidos permitían este análisis.

Finalmente, se debe destacar que la medida de no repetición concerniente a la elaboración del instructivo cumple con el objetivo de sentar un precedente transformador para evitar la concurrencia de los hechos expuestos. Bajo esta perspectiva, es

55. ONU, Comité de Derechos Económicos, Sociales y Culturales, Observación General n. ${ }^{\circ} 14$. El derecho al disfrute del más alto nivel posible de salud, 11 de agosto de 2000, E/C.12/2000/4. 
novedoso el esquema argumentativo que el juez Ávila presenta para sustentar que persistieron afectaciones a los derechos de la accionante por varios años sin ser reparados adecuadamente por los jueces que conocieron la apelación de la acción de protección. En atención de aquello, una observación que podría pasar desapercibida es que propiamente no se revisa la sentencia de instancia, sino que la CCE en realidad dicta una decisión de reemplazo a la que emitieron los jueces ordinarios. En el futuro, un rol del Pleno de la CCE debería orientarse a pormenorizar cuáles son las fallas concretas en la tramitación de las garantías jurisdiccionales a fin de corregirlas y evitar su repetición.

Todo lo anteriormente expuesto da cuenta de que las decisiones de la CCE en cuanto a su facultad de revisión no se vinculan únicamente a los jueces sino a las entidades que conforman el sector público, como en caso sucedió con el Ministerio de Salud y el IESS. Esta nueva perspectiva permite identificar que el máximo organismo de control, interpretación y protección de derechos no es un mero observador de la actividad de los jueces, sino un verdadero garante de derechos.

\section{BIBLIOGRAFÍA}

Agudelo, Carlos Alberto. "Diálogos internos en la Corte Constitucional Colombiana sobre el modus operandi en la selección de tutela entre la informalidad, la política y la academia". Jurídicas (Universidad de Caldas) 7, n. ${ }^{\circ} 2$ (2010): 53-78. https://bit.ly/3g07Zsi.

Aguirre, Pamela. "El valor de la jurisprudencia dentro del Ordenamiento Constitucional Ecuatoriano ¿Cambio de paradigma?”. Iurisprudentia Revista da Facultade de Direito D AJES 6, n. ${ }^{o} 12$ (2017): 111-36. https://bit.ly/3g07Zsi.

—, y PabloAlarcón. "El Estándar de la Reparación Integral en la Jurisprudencia de la Corte Constitucional". FORO: Revista de Derecho, n. ${ }^{\circ} 30$ (2018): 121-43. doi: 10.32719/26312484.2018.30.8.

Andrade, Karla. “La acción de protección en la jurisprudencia de la Corte”. En Manual de justicia constitucional ecuatoriana, editado por Jorge Benavides Ordóñez y Jhoel Escudero Soliz, 111-38. Quito: Corte Constitucional / Centro de Estudios y Difusión, 2013). https:// bit.ly/387KgDZ.

Atienza, Manuel, Curso de Argumentación Jurídica (Madrid: Trotta, 2013).

Ávila, Ramiro. La utopía del oprimido: los derechos de la naturaleza y el buen vivir en el pensamiento crítico, el derecho y la literatura. Ciudad de México: Akal, 2019.

Beltrán, Ramón. "La tópica jurídica y su vinculación argumentativa con el precedente y la jurisprudencia". Revista de Derecho de la Pontificia Universidad Católica de Valparaíso, n. 39 (2012): 587-606. doi: 10.4067/S0718-68512012000200021.

Córdova, Paúl. Derecho Procesal Constitucional. Quito: Corporación de Estudios y Publicaciones, 2016. 
Corte IDH. "Sentencia de 21 de julio de 1989 (Excepciones Preliminares, Fondo, Reparaciones y Costas)". Caso Velázquez Rodríguez vs. Honduras. 21 de julio de 1989. https://bit. ly/2ZhL5FV.

—. "Sentencia de 1 de julio de 2016 (Excepciones Preliminares, Fondo, Reparaciones y Costas)". Caso de las Masacres de Ituango vs. Colombia. https://bit.ly/3dLxCf7.

—. "Sentencia de 8 de marzo de 2018 (Excepciones Preliminares, Fondo, Reparaciones y Costas)". Caso Poblete Vilches vs. Ecuador, 8 de marzo de 2018. https://bit.ly/3dGIHOi.

Currea-Lugo, Víctor. La salud como derecho humano. Bilbao: Universidad Deusto, 2005.

Ecuador. Corte Constitucional. "Sentencia 001-PJO-CC". Caso n. 0999-09-JP. 22 de diciembre de 2010.

—. "Sentencia 001-14-PJO-CC". Caso n. ${ }^{\circ}$ 0067-11-JD. 23 de abril de 2014.

—. "Sentencia 016-16-SEP-CC". Caso n. ${ }^{\circ}$ 2014-12-EP. 13 de enero de 2016.

—. "Sentencia 184-18-SEP-CC". Caso n. ${ }^{\circ}$ 1692-12-EP. 29 de mayo de 2018.

—. "Sentencia 11-19-CN/19". Caso n. ${ }^{\circ} 11-19-C N / 19.12$ de junio de 2019.

—. "Sentencia 904-12-JP/19". Caso n. ${ }^{\circ}$ 904-12-JP. 13 de diciembre de 2019.

—. "Sentencia 159-11-JH/10". Caso n. ${ }^{\circ}$ 159-11-JH. 26 de noviembre de 2019.

—. "Sentencia 282-13-JP/19". Caso n. ${ }^{\circ}$ 282-13-JP. 4 de septiembre de 2019.

Ecuador. Constitución de la República del Ecuador. Registro Oficial 449, 20 de octubre de 2008.

Ecuador. Ley Orgánica de Garantías Jurisdiccionales y Control Constitucional. Registro Oficial, Suplemento. 22 de octubre de 2019.

Ecuador. Reglamento de Sustanciación de Procesos de Competencia de la Corte Constitucional. Registro Oficial, Suplemento 613. 22 de octubre de 2015, art. 25.

Galindo, Ernesto. Argumentación Jurídica: Técnicas de Argumentación del Abogado y del Juez. Ciudad de México: Porrúa, 2013.

Goberna-Tricas, Josefina. "Violencia obstétrica: aproximación al concepto y debate en relación a la terminología empleada". MUSAS. Revista de Investigación en Mujer, Salud y Sociedad 4, n. ${ }^{\circ} 2$ (2019): 26-36. doi: 10.1344/musas2019.vol4.num2.2.

Grijalva, Agustín. Constitucionalismo en Ecuador. Quito: Corte Constitucional para el Período de Transición, 2011.

Kelsen, Hans. Teoría Pura del Derecho. Traducido por Roberto Verengo. Ciudad de México: Porrúa, 2009.

Montaña, Juan. “Aproximación a los elementos básicos de la acción de protección”. En Apuntes de derecho procesal constitucional, editado por Juan Montaña Pinto y Angélica Porras Velasco, 135-56. Quito: Corte Constitucional para el Período de Transición / Centro de Estudios de Difusión del Derecho Constitucional Ecuatoriano, 2012.

ONU. Comité de Derechos Económicos, Sociales y Culturales, Observación General $n .^{\circ}$ 14. El derecho al disgrute del más alto nivel posible de salud. 11 de agosto de 2000. E/C.12/2000/4. 
- Observación General n. ${ }^{\circ}$ 19. El derecho a la seguridad social artículo 9. 4 de febrero de 2018. E/C.12/GC/19.

Pazmiño, Patricio. "El derecho a la salud y la especial protección de las personas con VIH. Desarrollo jurisprudencial y desafíos del acceso directo, progresividad y la reparación". Oficina del Alto Comisionado para los Derechos Humanos. 13 de febrero de 2019. https:// bit.ly/3dEsERc.

Rodríguez Manzo, Graciela, Iván Báez, Marcela Talamás y Miguel Pulido. Responsabilidady reparación en enfoque de Derechos Humanos (Ciudad de México: Comisión de Derechos Humanos del Distrito Federal / Universidad Iberoamericana, 2007).

Salem, Catalina. "Los conflictos de legalidad y los conflictos de constitucionalidad como garantía institucional de la jurisdicción constitucional". Revista de Derecho (Valdivia) 31, n. ${ }^{\circ}$ 2 (2018): 135-54. https://bit.ly/3fZslSz.

Serrano, Sandra, y Luis Vázquez. "Los principios de universalidad, interdependencia, indivisibilidad y progresividad. Apuntes para su aplicación práctica”. Biblioteca Jurídica Virtual del Instituto de Investigaciones Jurídicas de la UNAM. https://bit.ly/3g1U7xI.

Suárez, Emilio. "Distorsiones del sistema de selección y revisión de sentencias de la Corte Constitucional Ecuatoriana". Programa Maestría en Derecho, Mención en Derecho Constitucional, Universidad Andina Simón Bolívar, Sede Ecuador, 2015. https://bit.ly/2VqqUES.

Trujillo, Julio César. Constitucionalismo contemporáneo. Teoría, procesos, procedimientos y retos. Quito: Corporación Editora Nacional / Universidad Andina Simón Bolívar, Sede Ecuador, 2013. 